\title{
Mitarbeiter dieses Bandes
}

BORCK, Karl Heinz, geb. 1923, Dr. phil., Professor an der Universität Hamburg; zahlreiche Veröffentlichungen zur deutschen Literatur des Mittelalters.

CORKHILL, Alan, geb. 1948, Ph.D., Dozent an der Universität von Queensland (Brisbane); Publikationen zur deutschen Literatur des 19. und 20. Jahrhunderts.

DELBRÜCK, Hansgerd, geb. 1941, Dr, phil., Professor an der Victoria-Universität-Wellington; Veröffentlichungen zur deutschen Literatur des 18. und 19. Jahrhunderts.

FISHER, Rodney, geb. 1941, Ph.D., Dozent an der Universität von Canterbury (Christchurch); Veröffentlichungen zur deutschen Literatur des Mittelalters.

GRANT, Alyth, geb. 1946, M.A., Dozentin an der Universität von Otago (Dunedin); Veröffentlichungen zur Frauenliteratur.

HILLMAN, Roger, geb. 1946, Ph.D., Dozent an der Australian National University (Canberra); Publikationen zur deutschen Literatur des 19. und 20. Jahrhunderts.

OBERMAYER, August, geb. 1940, Dr. phil., Professor an der Universität von Otago (Dunedin); zahlreiche Publikationen zur deutschen, österreichischen und schweizerischen Literatur des 19. und 20. Jahrhunderts

TISCH, Johannes Hermann, geb. 1929, Dr. phil., Professor an der Universitäi von Tasmanien (Hobart); zahlreiche Veröffentlichungen zur europäischen Literatur des 17. und 18. Jahrhunderts.

WILPERT, Gero von, geb. 1933, Ph.D., Professor an der Universität von Sydney; zahlreiche Publikationen zur deutschen Literatur des 18., 19. und 20. Jahrhunderts, Verfasser und Herausgeber literarischer Nachschlagewerke. 